\title{
Ruanjian Sanjie Decoction Induces Apoptosis in Triple-negative Breast Cancer Cells by Downregulating cIAP1/2 and XIAP
}

Xiumei Zhao ( zxmmlg@163.com )

Centre for Research and Development of Anti Tumor drugs

\section{Tongxing Wang}

GeneNet Pharmaceuticals Co. Ltd. https://orcid.org/0000-0002-4008-7230

Qiang Jia

GeneNet Pharmaceuticals Co. Ltd.

\section{Luyao Wang}

Centre for Research and Development of Anti Tumor drugs

\section{Cheng Tan}

Centre for Research and Development of Anti Tumor drugs

\section{Hongsheng Shen}

Centre for Research and Development of Anti Tumor drugs

\section{Pengcheng Shi}

Centre for Research and Development of Anti Tumor drugs

\section{Lei Liu}

Tianjin Institute of Medical and Pharmaceutical Sciences

\section{Wenchang $\mathrm{Li}$}

Centre for Research and Development of Anti Tumor drugs

\section{Yunhui Hu}

GeneNet Pharmaceuticals Co. Ltd.

Hongbin Liu

Centre for Research and Development of Anti Tumor drugs

\section{Research}

Keywords: Ruanjian Sanjie decoction, inhibitor of apoptosis proteins, breast cancer, network pharmacology, mechanism of action

Posted Date: October 30th, 2020

DOl: https://doi.org/10.21203/rs.3.rs-97493/v1 
License: (c) (i) This work is licensed under a Creative Commons Attribution 4.0 International License. Read Full License 


\section{Abstract}

Background: Traditional Chinese medicine (TCM) comprises a unique theoretical system developed over thousands of years. The previous study reported that Ruanjian Sanjie (RJSJ) exerts anti-tumor effects by inducing cell apoptosis. However, the mechanism is not clear.

Methods: In this study, we investigated the possible mechanism by the strategy of combining network pharmacology analysis with experiment (in vitro and in vivo). First, four kinds of breast cancer cell lines were used to conduct proliferation, apoptosis and cell cycle analysis. Secondly, to study pathophysiological processes of breast cancer at the molecular network level, we for the first time constructed an "integrated apoptosis module network of breast cancer" by assembling the regulatory relationships of canonical apoptosis signaling pathways. Through the strategy of combining network analysis and experiments, we analyzed the main mechanism of RJSJ in breast cancer and screened out the core genes. We further studied the inhibitory effect of RJSJ combined with carboplatin (CBP) in vivo. Finally, the synergistic effect of RJSJ and CBP were analyzed and the potential active components in RJSJ were predicted.

Results: This study demonstrated that RJSJ could significantly inhibit breast cancer cell proliferation and induce apoptosis in a concentration-dependent manner. The primary mechanism of RJSJ in the treatment of breast cancer was pro-apoptotic. The core apoptosis genes regulated by RJSJ were clAP1/2 and XIAP. We also found that RJSJ in combination with CBP tended to synergistically induce apoptosis, which might mainly be achieved through the regulation of multiple targets and pathways. Alexandrin (BX05, XKC02, SCG01), baicalin (BX22), guanosine (BX32), arjunglucoside I (XKC10) etc. were predicted as potential active components.

Conclusions: These findings provide the rationale for exploring the therapeutic effects of RJSJ against breast cancer and providing a bridge for the combined use of Chinese and Western medicine.

\section{Background}

Breast cancer is the most common cancer and the second leading cause of death among women worldwide ${ }^{1}$. Based on the expression of estrogen receptor, progesterone receptor, human epidermal growth factor receptor 2, and Ki-67, breast cancers are divided into four subtypes: luminal A, luminal B, human epidermal growth factor receptor 2-enriched, and triple-negative breast cancer (TNBC). TNBC is the most aggressive subtype, and it is not sensitive to hormone and targeted therapy. Chemotherapy remains the standard treatment for TNBC because of the lack of targeted therapies ${ }^{2,3}$. Therefore, there is an urgent need to deepen our understanding of the pathogenesis of breast cancer and identify new treatment strategies.

The characteristics and advantages of traditional Chinese medicine (TCM) in the prevention and treatment of complex diseases, such as cancers, cardiovascular diseases, infectious diseases, metabolic diseases, and neurodegenerative diseases, have attracted global attention, and such treatments have 
been illustrated to ameliorate symptoms, improve patient quality of life, and prolong survival ${ }^{4-9}$. In 2018 , the WHO International Classification of Diseases listed traditional medicines, including TCM, in its classification system for the first time ${ }^{10}$. TCM, an important component of complementary and alternative medicine, has gradually developed its own unique system of theories, diagnostics, and therapies over thousands of years. At present, TCM has become an increasingly attractive source of novel therapeutic agents with significant roles in preventing and treating cancer ${ }^{11}$. TCMs can alleviate the side effects of chemotherapy, enhance immunity, and kill cancer cells ${ }^{12}$. For example, Ru ai xiao is an experienced prescription developed by Professor Sun from more than 40 years of clinical practice, which plays a role in controlling the progress of breast cancer patients, improving the quality of life and prolonging survival to a certain extent.

Ruanjian Sanjie decoction (RJSJ) consists of four herbs, namely Ban xia (Pinellia ternata), Xia ku cao (Prunella vulgaris), Shan ci gu (Cremastra appendiculata), and Hai zao (Sargassum pallidum). It has been traditionally used for softening hard lumps and resolving hard tissue masses. Our previous study illustrated that RJSJ has definite anti-tumor effects in vitro and in vivo. Meanwhile, RJSJ is safe and well tolerated in mice ${ }^{13,14}$. Although RJSJ has been demonstrated to have good curative effects against breast cancer, the fundamental molecular mechanisms have not been systematically explored. The bioactive compounds, potential targets, and related pathways of RJSJ remain unknown. The advancement of analytical tools including systems biology ${ }^{15}$, network biology ${ }^{16}$, and network pharmacology 17,18 potentially offer attractive strategies for elucidating the intricate and holistic mechanisms of Chinese herbal formulas.

In this study, we investigated the pro-apoptotic effects of RJSJ in a series of different breast cancer cell lines, including TNBC cell lines. The results suggested that the anti-tumor effect of RJSJ is mainly related to the induction of apoptosis. TCM-based network pharmacology analysis and experimental verification were employed to identify and verify the core targets of RJSJ in inducing apoptosis in breast cancer. In addition, when chemotherapy was combined with RJSJ, synergistic pro-apoptotic effects were observed, which might occur through multiple common or different targets in the "integrated apoptosis module network of breast cancer." Further, the potential active components in RJSJ that may play an important role in regulating apoptosis were predicted.

\section{Methods}

\section{Preparation of RJSJ}

All herbs in the RJSJ formula were obtained from Tianjin Zhong Xin Pharmaceutical Group Corporation Ltd. (Tianjin, China). RJSJ was prepared as a lyophilized dry powder of hot water extracts as described previously ${ }^{13}$. The composition was as follows: Pinellia ternate $(300 \mathrm{~g})$, Prunella vulgaris $(300 \mathrm{~g})$, Cremastra appendiculata $(200 \mathrm{~g})$, and Sargassum pallidum $(200 \mathrm{~g})$. Herbs were soaked for $0.5 \mathrm{~h}$ and extracted twice with 10 - and 8 -fold volumes of water at $100^{\circ} \mathrm{C}$ for $30 \mathrm{~min}$ each. The herbal broths were 
combined and filtered. Then, the filtrate was centrifuged twice at 12,000 rpm for $30 \mathrm{~min}$. The supernatants were mixed with an equal volume of ethanol, maintained at $4{ }^{\circ} \mathrm{C}$ overnight, and centrifuged at 12,000 rpm for $30 \mathrm{~min}$. The resultant supernatants were lyophilized, weighed, and stored at $-40^{\circ} \mathrm{C}$ until use. The lyophilized dry powder was dissolved in RPMI-1640 medium (Invitrogen; Thermo Fisher Scientific, Inc., Waltham, MA, USA) at the desired concentration and filtered through $0.22-\mu \mathrm{m}$ sterilization filters before use ${ }^{13,19}$

\section{Cell lines and mice}

The human breast cancer cell lines MDA-MB-231, MCF-7, Cal-51, and SKBR-3 were obtained from the American Type Culture Collection (Manassas, VA, USA) through the Cell Resource Center of the Tianjin Cancer Hospital (Tianjin, China). The cells were cultured in RPMI-1640 medium supplemented with 10\% fetal calf serum and $2 \mathrm{mM}$ I-glutamine (Gibco; Thermo Fisher Scientific Inc.). Cells were incubated at $37^{\circ} \mathrm{C}$ in an atmosphere of $5 \% \mathrm{CO}_{2}$ and saturated humidity. BALB/c-nude mice (female; age, 4- 6 weeks; weight, 16-18 g) were obtained from Vital River Laboratories Co., Ltd. (Beijing, China). Animals were maintained at $21-24^{\circ} \mathrm{C}$ with an $8 \mathrm{~h} / 16 \mathrm{~h}$ light/dark cycle and allowed to feed ad libitum. All in vivo studies were performed in the Central Laboratory of Tianjin Institute of Medical and Pharmaceutical Sciences (Tianjin, China) and approved by the Committee on Ethics of the Tianjin Institute of Medical and Pharmaceutical Sciences.

\section{3-(4, 5-Dimethylthiazol-2-yl)-2, 5 diphenyl tetrazolium bromide (MTT) assay}

Cal-51 and SKBR-3 cells in the logarithmic growth phase were seeded in 96-well plates at a density of $3 \times$ $10^{3} /$ well. Adherent cells were treated with RJSJ at different concentrations for 48 or $72 \mathrm{~h}$ in a humidified $\mathrm{CO}_{2}$ incubator at $37^{\circ} \mathrm{C}$. Then, $20 \mu$ of MTT solution were added to each well, and the cells were incubated at $37^{\circ} \mathrm{C}$ for $4 \mathrm{~h}$. The medium was aspirated carefully. The formazan crystals were solubilized with $150 \mu$ l of dimethyl sulfoxide, and the absorbance was measured at $570 \mathrm{~nm}$ to calculate the inhibition rate using the following formula: inhibition rate $(\%$ of control $)=([1-$ absorbance of test sample]/absorbance of control) $\times 100 \%$. IC 50 was determined via linear regression analysis using the inhibition rate against the corresponding concentration.

\section{Cellular morphology assay}

MDA-MB-231, MCF-7, Cal-51, and SKBR-3 cells in the logarithmic growth phase were seeded in 12-well plates at a density of $5 \times 10^{4}-10 \times 10^{4} /$ well. Based on the results of the MTT assay and our previous studies ${ }^{13}$, adherent cells were treated with different concentrations of RJSJ for $48 \mathrm{~h}$. Changes in cellular morphology were monitored using a ZEISS Axio Observer inverted microscope (ZEISS, Germany).

\section{Cell apoptosis analysis}

Cal-51 and SKBR-3 cells in the logarithmic growth phase were seeded in 12-well plates at a density of 10 $\times 10^{4} /$ well. After treatment with different concentrations of RJSJ, the cells were collected and washed 
with PBS. Collected cells resuspended in $500 \mu \mathrm{l}$ of binding buffer. Then, $5 \mu \mathrm{l}$ of annexin V-FITC and $5 \mu$ l of PI (BD Biosciences, Franklin Lakes, NJ, USA) were added to the buffer, followed by incubation at room temperature for $15 \mathrm{~min}$ in the dark. Cell apoptosis was analyzed via flow cytometry (BD FACSCanto Il; BD Biosciences) within $1 \mathrm{~h}$.

\section{Cell cycle analysis}

MDA-MB-231, MCF-7, Cal-51, and SKBR-3 cells were seeded in 12-well plates and treated with different concentrations of RJSJ for $48 \mathrm{~h}$. After treatment, the cells were collected and washed with PBS. Then, the cells were fixed with ice-cold $70 \%$ ethanol solution at $4{ }^{\circ} \mathrm{C}$ for $24 \mathrm{~h}$. RNase A solution $(100 \mu \mathrm{l})$ was added, and cells were incubated for $30 \mathrm{~min}$ at $37^{\circ} \mathrm{C}$. Finally, $400 \mu \mathrm{l}$ of PI were added, followed by incubation for $15 \mathrm{~min}$ at room temperature. The DNA content was detected via flow cytometry.

\section{Animal studies}

MDA-MB-231 cells and BALB/c-nude mice were used to construct the tumor-bearing animal model. A total of $1 \times 10^{7}$ MDA-MB-231 cells were suspended in $0.1 \mathrm{ml}$ of PBS containing $50 \%$ Matrigel (BD Biosciences) and injected into the mammary fat pads of 4-6-week-old female nude mice. Tumor size was measured every other day in two dimensions using a caliper. When the diameter of the induration reached 3-4 mm, 24 mice were randomly divided into four groups according to the mode of administration: control (NS $0.2 \mathrm{ml} / 10 \mathrm{~g}$ via gavage), carboplatin (CBP, $0.1 \mathrm{ml} / 10 \mathrm{~g}, 60 \mathrm{mg} / \mathrm{kg}$ via intraperitoneal injection once), RJSJ ( $0.2 \mathrm{ml} / 10 \mathrm{~g}, 500 \mathrm{mg} / \mathrm{kg}$ via gavage every day), and combination groups ( $500 \mathrm{mg} / \mathrm{kg}$ RJSJ via gavage and $60 \mathrm{mg} / \mathrm{kg}$ CBP via intraperitoneal injection). Mice were monitored for 21 days following treatment, and the size of subcutaneous tumors was measured every 4 days using calipers to verify the effects of drugs. The mice were sacrificed 21 days after drug administration, and the tumors were collected for protein extraction. Tumor volumes were calculated using the following formula: $(V)=\left(\right.$ long diameter $\times$ short diameter $\left.{ }^{2}\right) / 2$. The inhibition rate was calculated as follows: inhibition rate $(\%)=\left(\left[1-V_{\text {experimental group }}\right] / V_{\text {control group }}\right) \times 100 \%$.

\section{Western blot analysis}

MDA-MB-231 and Cal-51 cells were collected after manipulation as described in section Cellular morphology assay, and tumor tissues were obtained as described in section Animal studies. These samples were lysed on ice for 30 min to isolate proteins. The protein concentration was determined using a BCA protein assay kit (Beijing Solabel Technology Co., Ltd.). Protein lysates $(20 \mu \mathrm{g})$ were loaded on $12 \%$ SDS-PAGE gels and transferred to PVDF membranes (Merck Millipore, USA). Membranes were blocked in $5 \%$ nonfat milk for $1 \mathrm{~h}$, washed with TBST buffer, and incubated with primary antibodies at $4{ }^{\circ} \mathrm{C}$ overnight. Next, the membranes were washed and incubated with a secondary antibody at room temperature for $1 \mathrm{~h}$, followed by washing and transfer to ECL solution (Merck Millipore). Protein bands were visualized and detected using GE ImageQuant LAS 500 (GE, Japan). The results were measured using ImageJ software. Antibodies against cellular inhibitor of apoptosis protein 1 (clAP1, YM3009), cellular inhibitor of apoptosis protein 2 (clAP2, YM1343, (Immunoway), and X-linked inhibitor of apoptosis protein (XIAP, WL03561, Wanlibio Inc.) were used for immunodetection. 


\section{$\mathrm{RT}^{\mathbf{2}}$ Profiler PCR Array gene expression analysis}

MCF-7 cells were used to conduct the PCR array (PAHS-012A) after treatment with $500 \mu \mathrm{g} / \mathrm{ml}$ RJSJ or PBS. Mature RNA was isolated using an RNA extraction kit according to the manufacturer's instructions. RNA quality was determined using a spectrophotometer and reverse-transcribed using a cDNA conversion kit. The cDNA was subjected to the real-time RT ${ }^{2}$ Profiler PCR Array (QIAGEN, Cat. no. PAHS-012Z) in combination with $\mathrm{RT}^{2}$ SYBR ${ }^{\circledR}$ Green qPCR Mastermix (Cat. no. 330529). $\mathrm{C}_{\mathrm{T}}$ values were exported to an Excel file to create a table. This table was then uploaded to http://www.qiagen.com/geneglobe. Samples were assigned to the control or test group. $\mathrm{C}_{\mathrm{T}}$ values were normalized via automatic selection from a full panel of reference genes. The data analysis web portal calculated the fold change using the $\Delta \Delta \mathrm{C}_{\mathrm{T}}$ method, in which $\Delta \mathrm{C}_{\mathrm{T}}$ was calculated between the gene of interest and the average of reference genes followed by $\Delta \Delta \mathrm{C}_{\mathrm{T}}$ calculations $\left(\Delta \mathrm{C}_{\mathrm{T}}\right.$ [test group) $-\Delta \mathrm{C}_{\mathrm{T}}$ [control group]). Fold change was then calculated using the $2^{\Delta \Delta C T}$ formula.

\section{Network pharmacology analysis}

(1) Molecular database construction. We collected the component information of the four herbs in the RJSJ prescription using the ETCM database ${ }^{20}$. The MetaCore (https://portal.genego.com/), MalaCards (www.malacards.org) ${ }^{21}$, and DisGeNET databases ${ }^{22}$ were used to collect the causal genes of breast cancer. To obtain the apoptosis-related gene set defined using Gene Ontology (GO) terms, we retrieved data from the UniProt database ${ }^{23}$ using the following search strategy: goa = apoptosis AND organism = "Homo sapiens (human) [9606]." In addition, the AmiGo 2 database ${ }^{24}$ was searched using the term "apoptosis," narrowed using "Genes and gene products associated with GO terms," and filtered using "Homo sapiens" as the organism. By integrating the aforementioned two parts, we obtained the apoptosis gene set. (2) Target fishing and analysis. The potential target data of components in RJSJ were obtained from the ETCM database ${ }^{20}$. In addition, to improve the reliability and accuracy of the subsequent data analysis, we also collected the known activity data of the ingredients from the ChEMBL 25 and PubChem databases ${ }^{26}$ and then integrated these data. Finally, the compound-target interaction network was constructed using Cytoscape v3.7.1 software ${ }^{27}$. For CBP, the target information was collected from the MetaCore (https://portal.genego.com/), ChEMBL ${ }^{25}$, PubChem ${ }^{26}$, and CTD databases

28. (3) Pathway enrichment analysis. The MetaCore database was used to conduct the disease and pathway enrichment analysis to identify pathways closely related to breast cancer. (4) Mechanism analysis. Based on the apoptosis pathways closely related to breast cancer, the "integrated apoptosis module network of breast cancer" was constructed. The potential targets of RJSJ or CBP and the causal genes of breast cancer were mapped to analyze the pro-apoptotic mechanism of RJSJ. (5) Hub nodes identification. The Cytoscape plugin cytoHubba ${ }^{29}$, which provides 11 topological analysis methods for ranking nodes by their network features, was used to identify important nodes from the "integrated apoptosis module network of breast cancer." By comprehensively considering the information of several topological parameters, the important nodes were selected manually by biology experts. (6) Active compounds identification. To screen out the potential active compounds with favorable physicochemical 
and pharmacokinetic properties, we obtained the solubility and absorption properties of molecules from the ETCM database ${ }^{20}$. ADMET solubility level $>1$ and ADMET absorption level $>1$ were used to screen the potential active compounds.

\section{Statistical analysis}

Statistical analysis was performed using SPSS version 17.0 (SPSS, Inc., Chicago, IL, USA). All data are presented as the mean \pm SD. One-way analysis of variance was used to determine statistically significant differences between groups. $\mathrm{P}<0.05$ was considered to indicate a statistically significant result.

\section{Results}

\section{RJSJ concentration-dependently inhibited breast cancer cell proliferation}

In previous studies, we obtained the $\mathrm{IC}_{50}$ values of RJSJ in MDA-MB-231 and MCF-7 cells as 0.578 and $0.635 \mathrm{mg} / \mathrm{ml}$, respectively ${ }^{13}$. We included Cal-51 and SKBR-3 cells in the analysis to further verify the growth-inhibitory effects of RJSJ. The results demonstrated that the $I_{50}$ values of RJSJ in Cal-51 and SKBR-3 cells were $0.865 \pm 0.071$ and $1.654 \pm 0.170 \mathrm{mg} / \mathrm{ml}$, respectively, after $48 \mathrm{~h}$ of exposure and 0.855 \pm 0.092 and $1.566 \pm 0.188 \mathrm{mg} / \mathrm{ml}$, respectively, after $72 \mathrm{~h}$ of exposure (Fig. $1 \mathrm{~A}-\mathrm{B}$ ). This study indicated that the viability of these two cell lines was markedly reduced by RJSJ in a concentration-dependent manner.

Using an inverted microscope, control MDA-MB-231, MCF-7, Cal-51, and SKBR-3 were demonstrated to adhere to the plate, and the adjacent cells fused into pieces. As the RJSJ concentration was increased, the density of cells declined, the cell space increased, and cells began to necrose, indicating that RJSJ inhibited cell growth remarkably (Fig. 1C).

\section{RJSJ induced breast cancer cell apoptosis}

The effect of RJSJ on apoptosis in MDA-MB-231 and MCF-7 cells was previously reported by our group 13. In this study, we conducted apoptosis assays using Cal-51 and SKBR-3 cells. The results indicated that RJSJ also induced apoptosis in these cell lines in a concentration-dependent manner (Fig. 2).

\section{Effect of RJSJ on the cell cycle distribution in breast cancer cells}

To explore whether the inhibition of cell proliferation by RJSJ was associated with cell cycle arrest, we examined the cell cycle distribution of MDA-MB-231, MCF-7, Cal-51, and SKBR-3 cells using flow cytometry to analyze the cellular DNA content. The results indicated that RJSJ did not induce cell cycle arrest (Fig. 3), suggesting that the anti-tumor effect of RJSJ is mainly related to the induction of apoptosis. 


\section{RJSJ induces apoptosis in breast cancer cells by downregulating clAP1/2 and XIAP}

From the ETCM database, we constructed the component dataset for RJSJ. The number of compounds in Ban xia, Xia ku cao, Shan ci gu, and Hai zao are 40, 39, 16, and 27 respectively (Table S1). In total, 276 targets corresponding to components in RJSJ were obtained from the public databases ChEMBL and PubChem. Meanwhile, 495 targets were obtained from the ETCM database. There were 89 overlapping targets in these two target sets (Table S2). The interaction information of "component and target" is presented in Table S3.

Disease biomarker networks were used to conduct the enrichment analysis for RJSJ-related targets (Fig. S1, Table S4). In agreement with the experimental results in the aforementioned cell lines, the enrichment analysis results also suggested that the regulatory mechanism of RJSJ in breast cancer mainly occurs through the induction of apoptosis.

To elucidate the potential pharmacological mechanisms, especially the apoptosis-related mechanisms of RJSJ in breast cancer, we collected breast cancer genes from the MetaCore, MalaCards ${ }^{21}$, and DisGeNET databases ${ }^{22}$ and apoptosis genes from the UniProt ${ }^{23}$ and AmiGO 2 databases ${ }^{24}$. In total, 3410 causal genes of breast cancer (Table S5) and 132 apoptosis-related genes were collected (Table S6). The "integrated apoptosis module network of breast cancer" was assembled by integrating canonical apoptosis signaling pathways related to breast cancer in MetaCore (Fig. 4A). Details about this process are presented in Fig. S3. Network topology parameters were calculated using the cytoHubba plugin ${ }^{29}$ to evaluate the importance of nodes in the network. Notably, the experimental targets of RJSJ validated in our previous study, namely Bcl-2, survivin, caspase-3, caspase-7, and caspase-9, were highly ranked in this list (Table S7), suggesting that the analysis and calculation could provide potential targets involved in RJSJ treatment that deserve experimental validation.

As forecasted using the aforementioned "integrated apoptosis module network of breast cancer" and demonstrated in our previous study, RJSJ obviously induced apoptosis in MCF-7 cells. To validate the regulatory effects of RJSJ on the core apoptotic targets, MCF-7 cells were used to conduct the $\mathrm{RT}^{2}$ Profiler PCR Array (PAHS-012A) after treatment with $500 \mu \mathrm{g} / \mathrm{ml} \mathrm{RJSJ}$ or PBS. RT ${ }^{2}$ Profiler PCR Arrays are highly reliable and sensitive gene expression profiling tools for analyzing focused panels of genes in signal transduction, biological processes, or disease research pathways using real-time PCR. The analysis identified 27 differentially expressed apoptotic genes (Table S8).

In the $\mathrm{RT}^{2}$ Profiler PCR results of MCF-7 cells without evaluating XIAP (BIRC4), ClAP1 (BIRC2, fold change $=-5.99)$ and cIAP2 $(B I R C 3$, fold change $=-4.18)$ were differentially regulated by RJSJ, which is consistent with the aforementioned result of the "integrated apoptosis module network of breast cancer" (Table S7, Fig. 4A) and which was partly validated by our previous study ${ }^{13}$. Additionally, the regulatory relationship in network also revealed the direct regulatory effects of cIAP1/2 and XIAP on caspases. As reported in the literature, cIAP1/2 and XIAP participate in anti-apoptotic mechanisms in various cancer 
cells including breast cancer cells ${ }^{30-32}$. clAP1/2 and XIAP can bind and effectively inhibit caspase-3, caspase-7, and caspase-9 ${ }^{33-36}$. Consequently, by comprehensively considering the aforementioned results, cIAP1/2 and XIAP were selected for further experiments. The protein expression of $\mathrm{cIAP} 1 / 2$ and XIAP was detected in two TNBC cell lines. The results indicated that RJSJ inhibited cIAP1/2 and XIAP expression in MDA-MB-231 and Cal-51 cells in a concentration-dependent manner (Fig. 4B).

\section{RJSJ in combination with CBP inhibited transplanted tumor growth in mice}

In previous studies, we found that RJSJ inhibited the growth of transplanted tumors, prolonged the survival of Ehrlich ascites carcinoma-bearing mice, and enhanced the effects of 5-fluorouracil and doxorubicin ${ }^{13}$. To confirm whether RJSJ has broad-spectrum synergistic, we further studied the inhibitory effect of RJSJ combined with CBP in vivo. The animal weight increased slowly in each group during the entire test period (Fig. 5), but no abnormalities regarding diet consumption, bowel movements, and behavior were noted, indicating that CBP and RJSJ were well tolerated (Fig. 5A). As expected, tumor growth was slower in the three treatment groups than in the control group (Fig. 5B). The tumor growth inhibition rates in the RJSJ, CBP, and combination groups were $26.45,79.43$, and $85.78 \%$, respectively. RJSJ or CBP alone downregulated CIAP1/2 and XIAP, and combined treatment led to further inhibition of their expression (Fig. 5C-D). These results suggest that combining RJSJ with chemotherapy can enhance the tumor response by further inducing apoptosis.

\section{Analysis of the potential synergistic mechanism of RJSJ and CBP in promoting apoptosis in breast cancer}

A total of 136 CBP targets (Table S9) were collected from the MetaCore, ChEMBL ${ }^{25}$, PubChem ${ }^{26}$, and CTD databases ${ }^{28}$, including 14 apoptosis-related genes. Disease biomarker networks were used to conduct the enrichment analysis for CBP targets in breast cancer (Fig. S2). The results suggested that the regulatory mechanism of $\mathrm{CBP}$ in breast cancer mainly involves the induction of apoptosis, which is in line with some published studies ${ }^{37,38}$. Based on the compounds in RJSJ, we obtained 682 targets, 33 of which were also targets of CBP, including five apoptosis-related genes (Fig. 6A).

To identify potential targets exerting pro-apoptotic effects on breast cancer, we mapped the targets of RJSJ and CBP onto the "integrated apoptosis module network of breast cancer" (Fig. 4A). In total, we screened out 63 targets, among which RJSJ and CBP had 42 and 31 targets, respectively, including 10 common targets (Fig. 6B). The apoptosis module network obviously illustrated that RJSJ and CBP could exert synergetic effects by regulating common targets such as AKT1, CASP3, IL6, and TP53 or different targets in apoptosis pathways related to breast cancer (Fig. 4A).

Based on the 63 targets and their interactions with CBP or compounds in RJSJ, we constructed the compound-target network to further clarify the mechanism (Fig. 6C). Overall, 72 compounds in RJSJ with the potential to induce apoptosis in the "integrated apoptosis module network of breast cancer" were 
identified (Table S10). We screened out 25 potential active compounds (4 in Ban xia, 12 in Xia ku cao, 2 in Shan ci gu, and 7 in Hai zao) with favorable physicochemical and pharmacokinetics properties (ADMET solubility level > 1 and ADMET absorption level >1).

Further, based on the compound-target network, 30 compounds from RJSJ with potential synergistic effects with CBP on apoptosis with 10 common targets (overlapping part of Fig. 6B) were further identified (Fig. 6D). Of these compounds, 13 had good solubility and absorption.

Based on the aforementioned analysis, apoptosis-related genes, such as IL6, CASP3, AKT1, and TP53, and other closely related genes, including NFKB1, HSPB1, JUN, and CDC42, were considered the most crucial targets for combined treatment with RJSJ and CBP. Correspondingly, alexandrin (BX05, XKC02, SCG01), a common compound in three herbs; baicalin (BX22) and guanosine (BX32) from Ban xia; and arjunglucoside I (XKC10), niga-ichigoside F1 (XKC25), niga-ichigoside F2 (XKC26), pruvuloside A (XKC28), pruvuloside $B(X K C 29)$, sericoside (XKC30), vulgarsaponin $A(X K C 35)$, and vulgarsaponin $B$ (XKC36) from Xia ku cao were considered as significant apoptosis-inducing components deserving of further experimental validation.

\section{Discussion}

In this study, experimental and network pharmacology strategies were combined to study the mechanism of action of RJSJ in breast cancer. Both the experimental data and results of network pharmacology analysis suggested that the induction of apoptosis may be the primary mechanism of the therapeutic effects of RJSJ on breast cancer, which is consistent with the experimental results of a previous study ${ }^{13}$. To elucidate the mechanism by which RJSJ induces apoptosis, we constructed an "integrated apoptosis module network of breast cancer" for the first time by assembling the regulatory relationships of canonical apoptosis signaling pathways that are closely related to breast cancer, which systematically concretized the phenotype of disease physiological processes at the molecular network level. The MetaCore database contains high-quality and high-reliability data obtained via manual curation from studies. The regulatory relationship between the nodes has clear mechanisms (such as binding, transcriptional regulation, and phosphorylation) and effects (activation and inhibition). Deepening our understanding of the role of apoptosis in the development of breast cancer has great significance. Meanwhile, the integrated network could illuminate the key regulatory genes and pathways and provide a theoretical basis for the development of low-toxicity, high-efficacy drugs, such as combination drugs and multi-target drugs ${ }^{39-41}$. This is an efficient strategy for studying the pathophysiological processes of diseases. Subsequently, the potential core apoptotic genes playing important roles in the pro-apoptotic effects of RJSJ on breast cancer cells were screened using integrating network analysis, regulatory relationships, and experimental data in MCF-7 cells. The potential targets of RJSJ, such as Bcl-2, survivin, caspase-3, caspase-7, caspase-9, cIAP1/2, XIAP, AKT (PKB), p53, AKT1, and Bax, were the top ranked genes. Intriguingly, genes such as Bcl-2, survivin, caspase-3, caspase-7, and caspase-9 might be the potential targets of RJSJ, as indicated by our previous study ${ }^{13}$. CIAP1/2 and XIAP can bind and effectively inhibit caspase-3, caspase-7, and caspase-9, which participate in the anti-apoptotic 
mechanisms in various cancer cells including breast cancer cells ${ }^{30-36}$. The influence of RJSJ on these proteins was verified both in vitro and in vivo, supporting the efficiency of this strategy. Other hub nodes, such as AKT (PKB), p53, AKT1, and Bax, may be potential targets of RJSJ, but additional studies are required for validation. Generally, the high-quality pathophysiological process network could deepen our understanding of disease physiological processes and provide valuable clues for the discovery of important drug targets.

TCM holds the unique advantages of multi-target and multi-pathway regulation in the treatment of complex diseases with high efficacy, low toxicity, and few side effects ${ }^{4-9}$. Previous research illustrated that RJSJ is safe and well tolerated, and it does not induce body weight loss, immune dysregulation, or myelosuppression in mice ${ }^{13}$. In recent years, the combination of Chinese and Western medicines has attracted increasing attention. Reasonable combinations of Chinese and Western medicines can lead to enhanced curative effects, reduced side effects, and lower drug doses ${ }^{42-45}$. However, improper combinations of Chinese and Western medicines will lead to reduced curative effects and greater side effects. CBP is a broad-spectrum anti-tumor drug that is widely used clinically. However, its side effects include myelosuppression, gastrointestinal reactions, and allergic reactions ${ }^{46}$. Using CBP as an example, we preliminarily studied the effect of RJSJ combined with Western medicine to provide evidence for clinical usage. The results revealed that the combination had no side effects, and the effects of the regimen on tumor volume were not significantly different from those of CBP alone. However, RJSJ and $\mathrm{CBP}$ in combination synergistically increased the expression of apoptotic proteins, which was consistent with the aforementioned experimental results at cellular level.

To elucidate the synergistic mechanism of RJSJ and CBP, we analyzed their targets at the molecular and network levels. The results illustrated that the synergistic effect of CBP and RJSJ was reflected in the regulation of the "apoptosis module network of breast cancer" and other pathways, such as those related with inflammatory responses (Table S4). The molecular network involved in cancer is extremely complex, and alternative pathways usually exist 47,48 . After blocking one specific signaling pathway, tumors can activate signal transduction through other pathways, which makes it difficult for single-target drugs to achieve good therapeutic effects ${ }^{47}$. In this study, by mapping the targets of RJSJ and CBP onto the "apoptosis module network of breast cancer," we clearly revealed that they have both common and unique targets. The combination of RJSJ and CBP could increase coverage for targets distributed across multiple apoptotic pathways. This may be the main principle of the synergistic effects of RJSJ and CBP on apoptosis in breast cancer cells.

To further elucidate the material basis of the induction of apoptosis by RJSJ in breast cancer cells, we predicted and screened the active components. We identified active components with potential proapoptotic effect in all four plants, which reflects their synergistic effects. This is consistent with the principles of formulating TCM prescriptions. According to the compatibility of "monarch, minister, adjuvant and guide," the entire prescription for a specific indication can enhance efficacy and reduce toxicity ${ }^{49}$. It has been reported that the four herbs in RJSJ inhibited tumor cell growth and induced 
apoptosis. For example, when human medullary thyroid carcinoma TT cells treated with Prunella vulgaris, mitochondrial membrane potential was significantly decreased, CYCS expression in mitochondria was increased, procaspase-3 expression was reduced, and activated caspase-3 expression was increased ${ }^{50}$. Fucoidin in Sargassum pallidum significantly inhibited the growth of HeLa cells and reduced Bcl-2 the expression ${ }^{51}$. The expression of Bcl-2 in SW579 thyroid cancer cells was effectively reduced by Cremastra appendiculata ${ }^{52}$. The water extract of Pinellia ternata strongly inhibited K562 cell proliferation, induced apoptosis, and blocked cell cycle progression at G0/G1 phase ${ }^{53}$. Concerning potential active compounds, such as, alexandrin, baicalin (BX22), guanosine (BX32), arjunglucoside I (XKC10), niga-ichigoside F1 (XKC25), niga-ichigoside F2 (XKC26), pruvuloside $A$ (XKC28), pruvuloside $B$ (XKC29), sericoside (XKC30), vulgarsaponin (XKC35), and vulgarsaponin $\mathrm{B}$ (XKC36) were considered potential active components that induced apoptosis, but further experimental validation is needed. Although some important results were obtained and preliminary understanding of the pro-apoptotic mechanism for RJSJ in breast cancer was achieved, additional experiments are required to further investigate the biological functions and mechanism of this decoction.

\section{Conclusions}

In conclusion, this study demonstrated that RJSJ could significantly inhibit breast cancer cell proliferation and induce apoptosis in a concentration-dependent manner. The primary mechanism of RJSJ in the treatment of breast cancer was the induction of apoptosis. Based on the "apoptosis module network of breast cancer," we screened and verified that RJSJ could induce apoptosis in breast cancer cells by regulating the core nodes CIAP1/2 and XIAP in vitro and in vivo. We also found that RJSJ in combination with CBP tended to synergistically induce apoptosis, which might mainly be achieved through the regulation of multiple targets and pathways in breast cancer apoptosis networks. Through the molecular network analysis of disease physiological processes, we can systematically understand disease physiological processes at the molecular level, which could lay the foundation for the development of effective, low-toxicity drugs. In addition, through network pharmacology technology, the complex mechanism and material basis of the RJSJ prescription were explained in a relatively clear manner, and its synergistic effect with CBP was preliminarily explained, providing directions for future research and experimental support for monotherapy or combination therapy with RJSJ in the clinical treatment of tumors. Therefore, the anti-tumor activities of RJSJ make it a promising TCM for malignancy. The results both significantly improve our understanding of breast cancer and clarify the mechanism of action of RJSJ, which should accelerate the application of TCM in modern medicine and promote the modernization of TCM.

\section{Abbreviations}

TCM, Traditional Chinese medicine; RJSJ, Ruanjian Sanjie decoction; CBP, carboplatin; MTT, 3-(4, 5dimethylthiazol-2-yl)-2, 5 diphenyl tetrazolium bromide; cIAP1, cellular inhibitor of apoptosis protein 1; cIAP2, cellular inhibitor of apoptosis protein 2; XIAP, X-linked inhibitor of apoptosis protein. 


\section{Declarations}

\section{Ethics approval and consent to participate}

Not applicable.

\section{Consent for publication}

Not applicable.

\section{Availability of data and materials}

All data generated or analysed during this study are included in this published article [and its supplementary information files].

\section{Competing interests}

The author reports no conflicts of interest in this work.

\section{Funding}

This work was supported by the scientific research project on integration of traditional Chinese and Western Medicine of Tianjin Health and family planning commission (No. 2017080).

\section{Author's contributions}

X.M.Z.* conceived and designed the study. Y.H.H.* and H.B.L.* coordinated technical support and funding. X.M.Z.* and T.X.W. wrote the manuscript. Q.J., L.Y.W., C.T., H.S.S., and P.C.S. performed the experiments and revised the manuscript critically for important intellectual content. L.L. and W.C.L. were responsible for the collection and analysis of the experimental data. All authors read and approved the final manuscript.

\section{Acknowledgments}

We are grateful to the scientific research project on integration of traditional Chinese and Western Medicine of Tianjin Health and family planning commission. We thank Joe Barber Jr., PhD, from Liwen Bianji, Edanz Editing China (www.liwenbianji.cn/ac), for editing the English text of a draft of this manuscript.

\section{Authors' information}

${ }^{1}$ Centre for Research and Development of Anti Tumor drugs, Tianjin Institute of Medical and Pharmaceutical Sciences, Tianjin 300020, P.R. China. ${ }^{2}$ The Third Department of Breast Cancer, Tianjin Medical University Cancer Institute and Hospital, Tianjin 300060, P.R. China. ${ }^{3}$ GeneNet Pharmaceuticals 
Co. Ltd., Tianjin 300410, P.R. China. ${ }^{4}$ Department of Neurosurgery, Tianjin Huanhu Hospital, Tianjin 300350, P.R. China

\section{References}

1. Waks AG, Winer EP. Breast Cancer Treatment: A Review. Jama. Jan 22 2019;321(3):288-300. doi:10.1001/jama.2018.19323

2. Gaynor N, Crown J, Collins DM. Immune checkpoint inhibitors: Key trials and an emerging role in breast cancer. Seminars in cancer biology. Jul 2 2020;doi:10.1016/j.semcancer.2020.06.016

3. Wein L, Luen SJ, Savas P, Salgado R, Loi S. Checkpoint blockade in the treatment of breast cancer: current status and future directions. British journal of cancer. Jul 2018;119(1):4-11. doi:10.1038/s41416-018-0126-6

4. Jia W, Wang L. Using Traditional Chinese Medicine to Treat Hepatocellular Carcinoma by Targeting Tumor Immunity. Evidence-based complementary and alternative medicine : eCAM. 2020;2020:9843486. doi:10.1155/2020/9843486

5. Guo W, Tan HY, Chen F, Wang N, Feng Y. Targeting Cancer Metabolism to Resensitize Chemotherapy: Potential Development of Cancer Chemosensitizers from Traditional Chinese Medicines. Cancers. Feb 10 2020;12(2)doi:10.3390/cancers12020404

6. Guo R, Luo X, Liu J, Liu L, Wang X, Lu H. Omics strategies decipher therapeutic discoveries of traditional Chinese medicine against different diseases at multiple layers molecular-level. Pharmacological research. Feb 2020;152:104627. doi:10.1016/j.phrs.2020.104627

7. Wang Y, Zhao H, Wang Q, et al. Chinese Herbal Medicine in Ameliorating Diabetic Kidney Disease via Activating Autophagy. Journal of diabetes research. 2019;2019:9030893. doi:10.1155/2019/9030893

8. Eng YS, Lee CH, Lee WC, Huang CC, Chang JS. Unraveling the Molecular Mechanism of Traditional Chinese Medicine: Formulas Against Acute Airway Viral Infections as Examples. Molecules (Basel, Switzerland). Sep 27 2019;24(19)doi:10.3390/molecules24193505

9. Jian X, Liu Y, Zhao Z, Zhao L, Wang D, Liu Q. The role of traditional Chinese medicine in the treatment of atherosclerosis through the regulation of macrophage activity. Biomedicine \& pharmacotherapy $=$ Biomedecine \& pharmacotherapie. Oct 2019;118:109375. doi:10.1016/j.biopha.2019.109375

10. Deng H, Liang S, Clive EA, Farhad S. Classification of interventions in Traditional Chinese Medicine. Journal of traditional Chinese medicine = Chung $i$ tsa chih ying wen pan. Apr 2018;38(2):315-320.

11. Ling CQ, Yue XQ, Ling C. Three advantages of using traditional Chinese medicine to prevent and treat tumor. Journal of integrative medicine. Jul 2014;12(4):331-5. doi:10.1016/S2095-4964(14)60038-8

12. Zhao Y, Yang A, Tu P, Hu Z. Anti-tumor effects of the American cockroach, Periplaneta americana. Chinese medicine. 2017;12:26. doi:10.1186/s13020-017-0149-6

13. Zhao X, Zhao J, Hu R, et al. Ruanjian Sanjie decoction exhibits antitumor activity by inducing cell apoptosis in breast cancer. Oncology letters. May 2017;13(5):3071-3079. doi:10.3892/ol.2017.5832 
14. Xiu-Mei Z, Yun-Hui HU, Hong-Sheng S, Xi-Jing LI, Wen-Chang LI, Ren-Jie HU. Influence of Ruanjian Sanjie prescription on pharmacokinetics of 5-fluorouracil in mice and its anti-tumor activity. Military Medical ences. 2018;42(10):749-752.

15. Kitano H. Systems biology: a brief overview. Science (New York, NY). Mar 1 2002;295(5560):1662-4. doi:10.1126/science.1069492

16. Barabasi AL, Oltvai ZN. Network biology: understanding the cell's functional organization. Nature reviews Genetics. Feb 2004;5(2):101-13. doi:10.1038/nrg1272

17. Hopkins AL. Network pharmacology. Nature biotechnology. Oct 2007;25(10):1110-1. doi:10.1038/nbt1007-1110

18. Hopkins AL. Network pharmacology: the next paradigm in drug discovery. Nature chemical biology. Nov 2008;4(11):682-90. doi:10.1038/nchembio.118

19. Hu B, An HM, Shen KP, et al. Liver Yin deficiency tonifying herbal extract induces apoptosis and cell senescence in Bel-7402 human hepatocarcinoma cells. Experimental and therapeutic medicine. Jan 2012;3(1):80-86. doi:10.3892/etm.2011.364

20. Xu HY, Zhang YQ, Liu ZM, et al. ETCM: an encyclopaedia of traditional Chinese medicine. Nucleic acids research. Jan 8 2019;47(D1):D976-D982. doi:10.1093/nar/gky987

21. Stelzer G, Rosen N, Plaschkes I, et al. The GeneCards Suite: From Gene Data Mining to Disease Genome Sequence Analyses. Current protocols in bioinformatics. Jun 20 2016;54:1.30.1-1.30.33. doi:10.1002/cpbi.5

22. Piñero J, Ramírez-Anguita JM, Saüch-Pitarch J, et al. The DisGeNET knowledge platform for disease genomics: 2019 update. Nucleic acids research. Jan 8 2020;48(D1):D845-d855. doi:10.1093/nar/gkz1021

23. UniProt Consortium T. UniProt: the universal protein knowledgebase. Nucleic acids research. Mar 16 2018;46(5):2699. doi:10.1093/nar/gky092

24. Ashburner M, Ball CA, Blake JA, et al. Gene ontology: tool for the unification of biology. The Gene Ontology Consortium. Nature genetics. May 2000;25(1):25-9. doi:10.1038/75556

25. Gaulton A, Hersey A, Nowotka M, et al. The ChEMBL database in 2017. Nucleic acids research. Jan 4 2017;45(D1):D945-D954. doi:10.1093/nar/gkw1074

26. Wang Y, Xiao J, Suzek TO, et al. PubChem's BioAssay Database. Nucleic acids research. Jan 2012;40(Database issue):D400-12. doi:10.1093/nar/gkr1132

27. Shannon P, Markiel A, Ozier O, et al. Cytoscape: a software environment for integrated models of biomolecular interaction networks. Genome research. Nov 2003;13(11):2498-504. doi:10.1101/gr.1239303

28. Davis AP, Grondin CJ, Johnson RJ, et al. The Comparative Toxicogenomics Database: update 2019. Nucleic acids research. Jan 8 2019;47(D1):D948-D954. doi:10.1093/nar/gky868

29. Chin $\mathrm{CH}$, Chen $\mathrm{SH}, \mathrm{Wu} \mathrm{HH}, \mathrm{Ho} \mathrm{CW}$, Ko MT, Lin CY. cytoHubba: identifying hub objects and subnetworks from complex interactome. BMC systems biology. 2014;8 Suppl 4(Suppl 4):S11. 
doi:10.1186/1752-0509-8-S4-S11

30. Pluta P, Jeziorski A, Cebula-Obrzut AP, Wierzbowska A, Piekarski J, Smolewski P. Expression of IAP family proteins and its clinical importance in breast cancer patients. Neoplasma. 2015;62(4):666-73. doi:10.4149/neo_2015_080

31. Majorini MT, Manenti G, Mano M, et al. clAP1 regulates the EGFR/Snai2 axis in triple-negative breast cancer cells. Cell death and differentiation. Dec 2018;25(12):2147-2164. doi:10.1038/s41418-0180100-0

32. Delbue D, Mendonça BS, Robaina MC, et al. Expression of nuclear XIAP associates with cell growth and drug resistance and confers poor prognosis in breast cancer. Biochimica et biophysica acta Molecular cell research. May 30 2020:118761. doi:10.1016/j.bbamcr.2020.118761

33. Gyrd-Hansen M, Meier P. IAPs: from caspase inhibitors to modulators of NF-kappaB, inflammation and cancer. Nature reviews Cancer. 2010;10(8):561-574.

34. Roy N, Deveraux QL, Takahashi R, Salvesen GS, Reed JC. The c-IAP-1 and c-IAP-2 proteins are direct inhibitors of specific caspases. The EMBO journal. 1997;16(23):6914-6925.

35. Srinivasula SM, Ashwell JD. IAPs: what's in a name? Molecular cell. 2008;30(2):123-135.

36. Vucic D, Stennicke HR, Pisabarro MT, Salvesen GS, Dixit VM. ML-IAP, a novel inhibitor of apoptosis that is preferentially expressed in human melanomas. Current biology : CB. 2000;10(21):1359-1366.

37. Deng X, Chen Y, Liu Z, Xu J. MiR-124-3p.1 Sensitizes Ovarian Cancer Cells to Mitochondrial Apoptosis Induced by Carboplatin. OncoTargets and therapy. 2020;13:5375-5386. doi:10.2147/ott.s242342

38. Wang SZ, Poore B, Alt J, et al. Unbiased Metabolic Profiling Predicts Sensitivity of High MYCExpressing Atypical Teratoid/Rhabdoid Tumors to Glutamine Inhibition with 6-Diazo-5-Oxo-LNorleucine. Clinical cancer research : an official journal of the American Association for Cancer Research. Oct 1 2019;25(19):5925-5936. doi:10.1158/1078-0432.ccr-19-0189

39. Chen ZF, Orvig C, Liang H. Multi-Target Metal-Based Anticancer Agents. Current topics in medicinal chemistry. Nov 20 2017;17(28):3131-3145. doi:10.2174/1568026617666171004155437

40. Zheng W, Zhao Y, Luo Q, Zhang Y, Wu K, Wang F. Multi-Targeted Anticancer Agents. Current topics in medicinal chemistry. Nov 20 2017;17(28):3084-3098. doi:10.2174/1568026617666170707124126

41. Zhang Z, Zhou L, Xie N, et al. Overcoming cancer therapeutic bottleneck by drug repurposing. Signal transduction and targeted therapy. Jul 2 2020;5(1):113. doi:10.1038/s41392-020-00213-8

42. Luo X, Ni X, Lin J, et al. The add-on effect of Chinese herbal medicine on COVID-19: A systematic review and meta-analysis. Phytomedicine : international journal of phytotherapy and phytopharmacology. Jul 11 2020:153282. doi:10.1016/j.phymed.2020.153282

43. Ding Y, Liu Y, Li H, et al. Chinese Medicines for Preventing and Treating Radiation-Induced Pulmonary Injury: Still a Long Way to Go. Frontiers in pharmacology. 2019;10:927. doi:10.3389/fphar.2019.00927 
44. Huang Y, Zhu J, Lin X, Hong Y, Feng Y, Shen L. Potential of Fatty Oils from Traditional Chinese Medicine in Cancer Therapy: A Review for Phytochemical, Pharmacological and Clinical Studies. The American journal of Chinese medicine. 2019;47(4):727-750. doi:10.1142/s0192415x19500381

45. Mao D, Feng L, Huang S, Zhang S, Peng W, Zhang S. Meta-Analysis of Xihuang Pill Efficacy When Combined with Chemotherapy for Treatment of Breast Cancer. Evidence-based complementary and alternative medicine : eCAM. 2019;2019:3502460. doi:10.1155/2019/3502460

46. Oun R, Moussa YE, Wheate NJ. The side effects of platinum-based chemotherapy drugs: a review for chemists. Dalton transactions (Cambridge, England : 2003). May 15 2018;47(19):6645-6653. doi:10.1039/c8dt00838h

47. Mei XL, Wei FL, Jia LL, Ji YZ. An alternative pathway for cellular protection in BRAF inhibitor resistance in aggressive melanoma type skin cancer. Chemico-biological interactions. May 25 2020;323:109061. doi:10.1016/j.cbi.2020.109061

48. Nouri Z, Fakhri S, Nouri K, Wallace CE, Farzaei MH, Bishayee A. Targeting Multiple Signaling Pathways in Cancer: The Rutin Therapeutic Approach. Cancers. Aug 14 2020;12(8)doi:10.3390/cancers12082276

49. Li WX, Tang YP, Shi XQ, Guo JM, Liu P, Duan JA. [Research on Chinese medicine pairs (IV)-Their compatibility effects]. Zhongguo Zhong yao za zhi = Zhongguo zhongyao zazhi = China journal of Chinese materia medica. Dec 2013;38(24):4203-7.

50. XIONG Y, ZHAO M, TAN J-b, YANG Z, ZHANG W-j, YANG X-f. Effects and mechanism of Spica Prunellae on proliferation and apoptosis of human medullary thyroid carcinoma TT cells. China Journal of Traditional Chinese Medicine and Pharmacy. 2018;33(08):3379-3384.

51. Chenxi HU, Baoheng X, Jian L, et al. Preliminary study on anti-tumor activity of fucoidin of Sargassum fusiforme. Drug Evaluation Research. 2018;41(09):1581-1588.

52. Zhifan YU, Yinghua L, Juncai X, Mingwei W, Surgery DO. Effect of cremastra appendiculata makino on proliferation and apoptosis of thyroid cancer SW579 cells. Oncology Progress. 2018;16(10):1292$1294+1298$.

53. Meng WU, Bin C. Primary study on apoptosis of K562cells in vitro induced by water extract of Pinellia ternate(Thunb)Breit. Journal of Clinical Hematology. 2014;27(06):983-986.

\section{Supplementary Information}

Figure S1. Top 10 enriched disease biomarker networks of the targets of Ruanjian Sanjie;

Figure S2. Top 10 enriched disease biomarker networks of the targets of carboplatin;

Figure S3. Flowchart for construction of the apoptosis module network of breast cancer using integrating canonical apoptosis signaling pathways;

Table S1. Compound information of the four herbs of Ruanjian Sanjie; 
Table S2. Known and predicted targets data;

Table S3. Known and predicted compound-targets interaction data;

Table S4. Enrichment analysis of disease biomarker networks of the targets of Ruanjian Sanjie and carboplatin;

Table S5. Breast cancer gene set;

Table S6. Apoptosis gene set;

Table S7. cytoHubba score calculated using 11 methods;

Table S8 PCR results of gene expression following Ruanjian Sanjie treatment in MCF-7 cells;

Table S9. Target set of carboplatin;

Table S10. Potential components in Ruanjian Sanjie exerting pro-apoptotic effect on breast cancer cells.

\section{Figures}


A

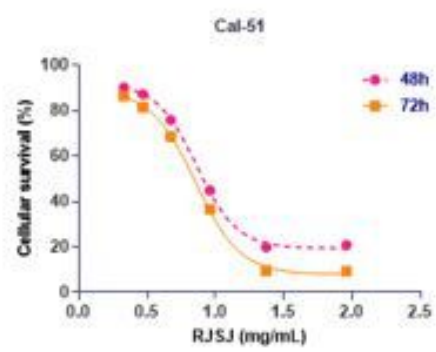

SKeR-3

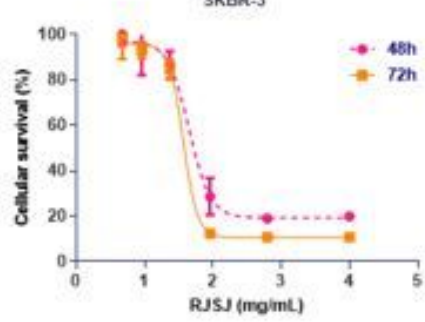

B

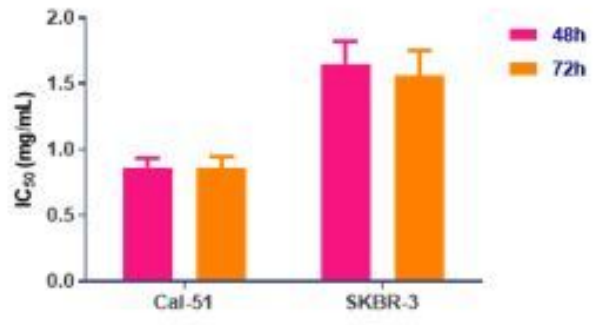

C

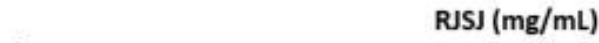

MDA-MB-231

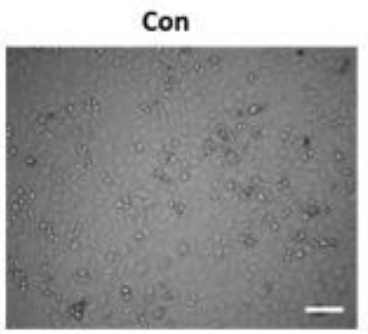

0.5

1.0
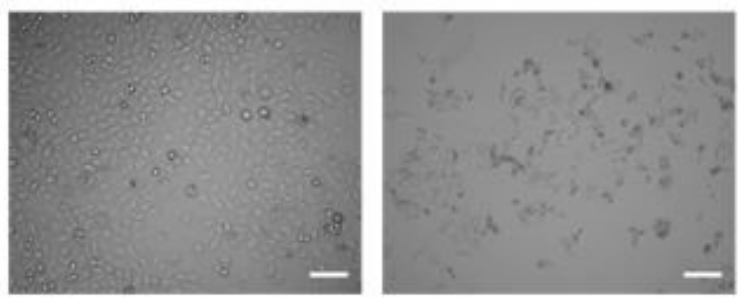

1.5

Con

1.0

1.5
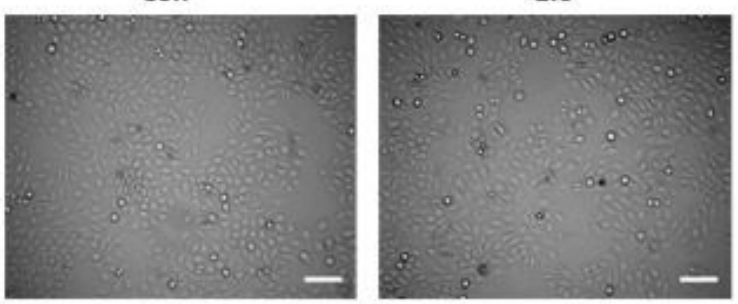

0.5

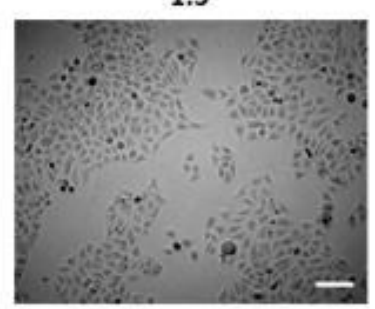

1.0
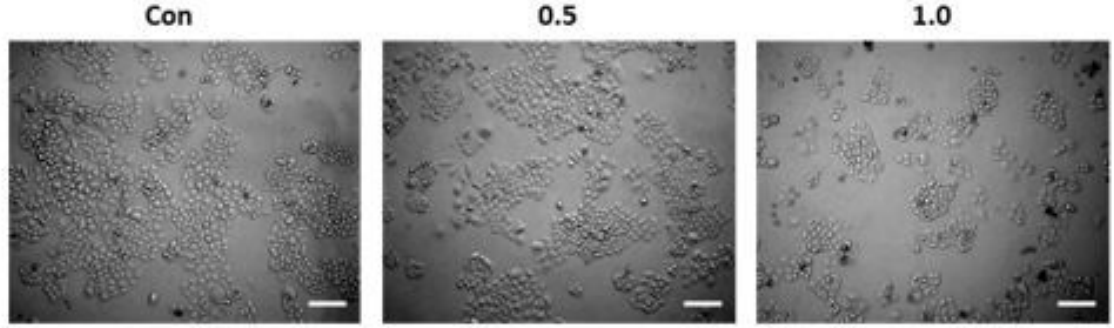

1.5

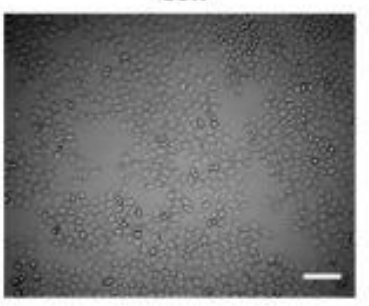

1.0
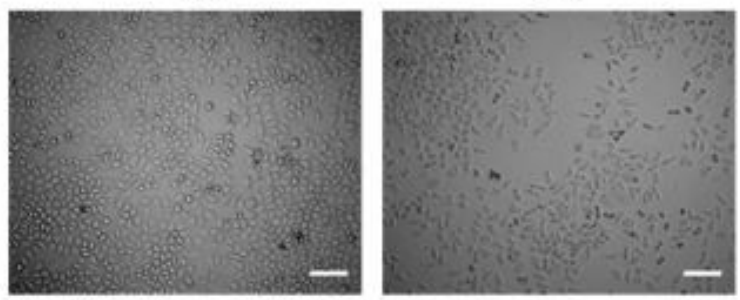

1.5

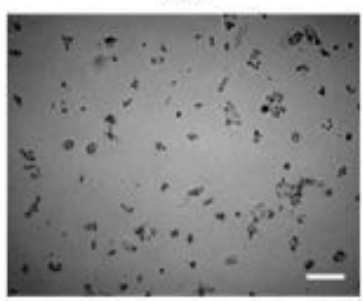

2.0

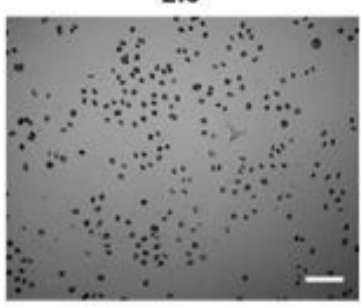

$400 \mu \mathrm{m}$

\section{Figure 1}

Ruanjian Sanjie decoction (RJSJ) inhibited the proliferation of breast cancer cells. (A) Concentrationresponse curves used to calculate the IC50 of RJSJ in Cal-51 and SKBR-3 cells. (B) IC50 values of RJSJ in Cal-51 and SKBR-3 cells at 48 and $72 \mathrm{~h}$. (C) The morphological changes of cells treated with RJSJ were observed using an inverted microscope $(\times 200)$. 

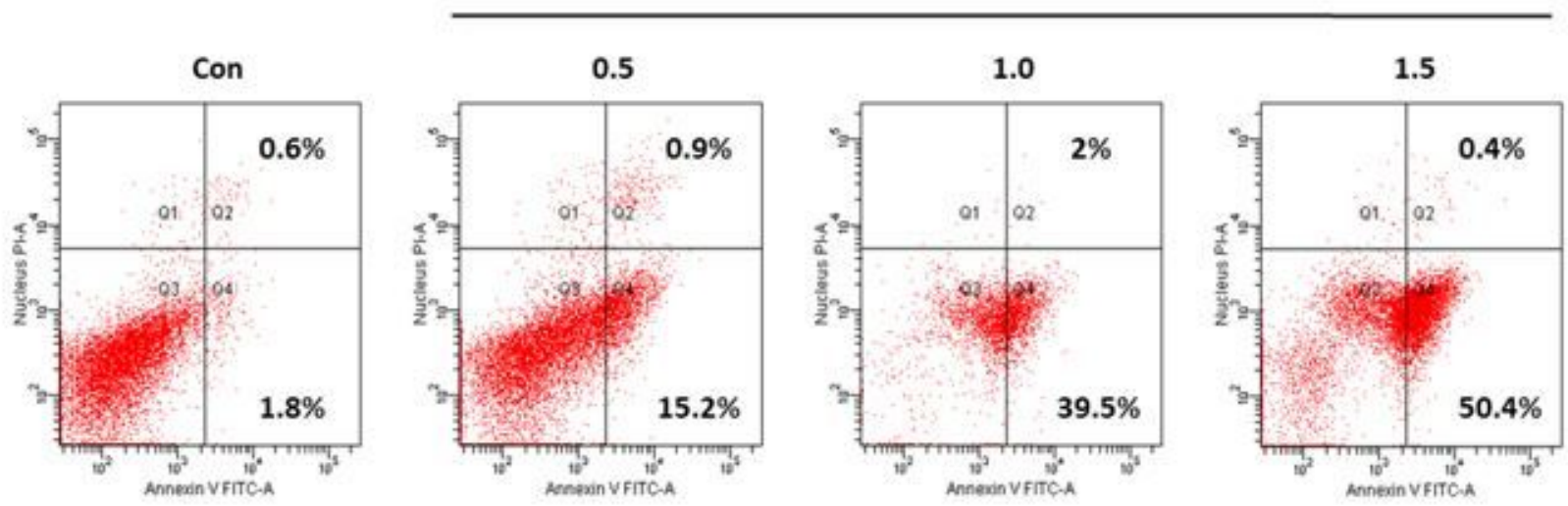

Cal-51
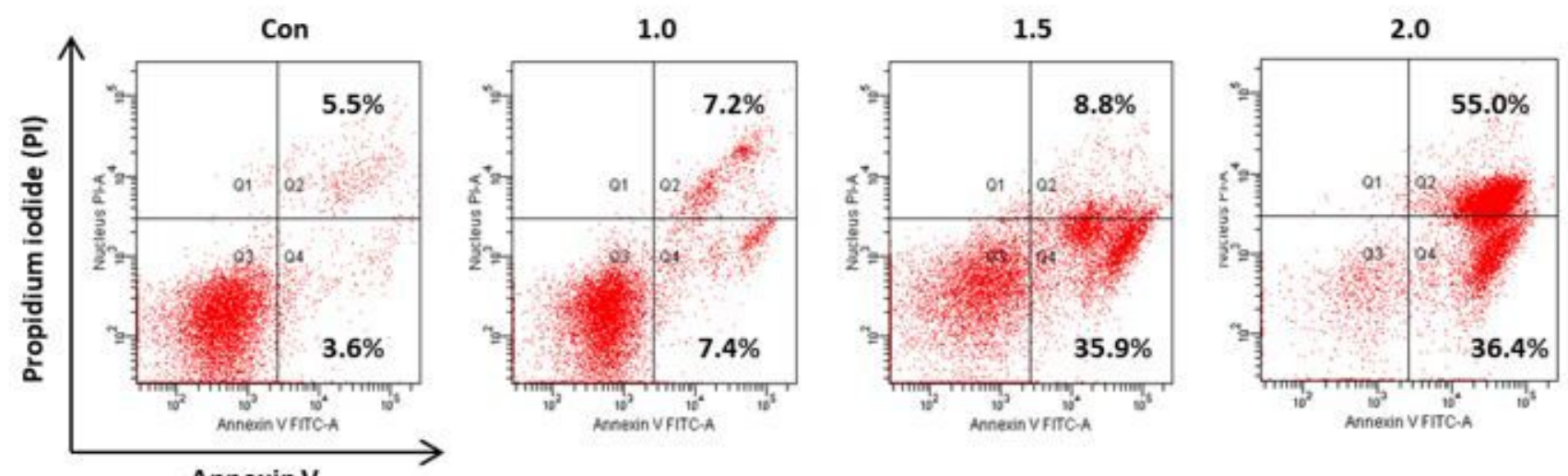

SKBR-3

B

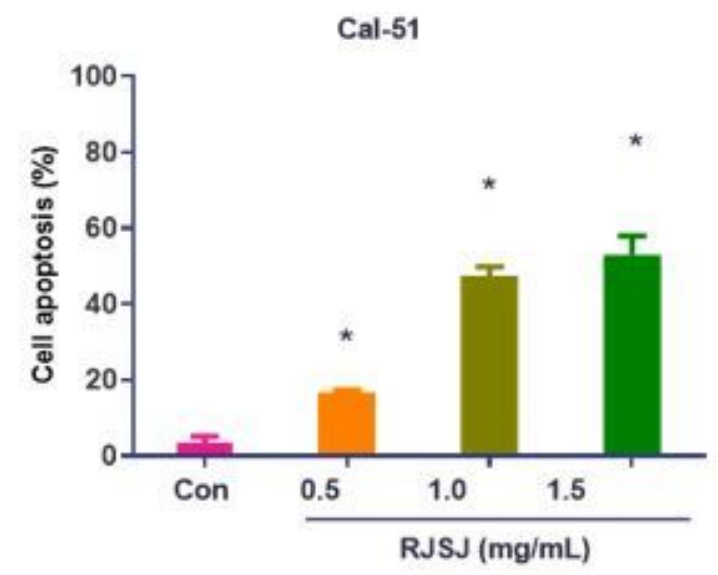

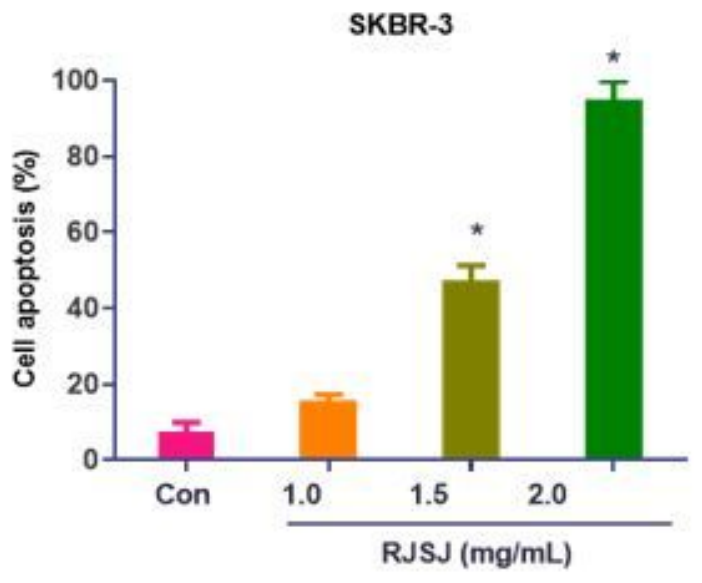

Figure 2

Ruanjian Sanjie (RJSJ) induced apoptosis in breast cancer cells. (A) Analysis of apoptosis in Cal-51 and SKBR-3 cells using flow cytometry after annexin V/PI double staining. (B) The percentages of apoptotic Cal-51 cells and SKBR-3 cells. Cells were treated with various concentrations of RJSJ or a vehicle control for $48 \mathrm{~h}$. Apoptosis was analyzed via flow cytometry after annexin V/PI double staining. The percentages of apoptotic cells are presented as the mean $\pm S D, * P<0.05 \mathrm{vs}$. control. 

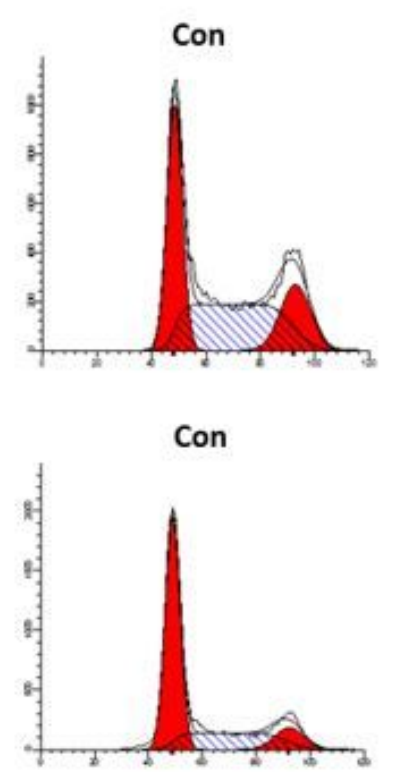

Con

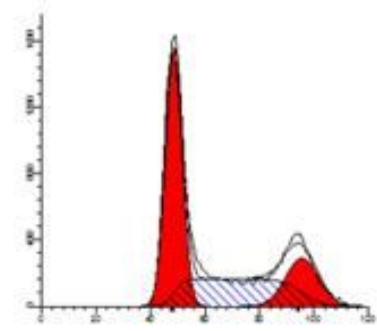

Con

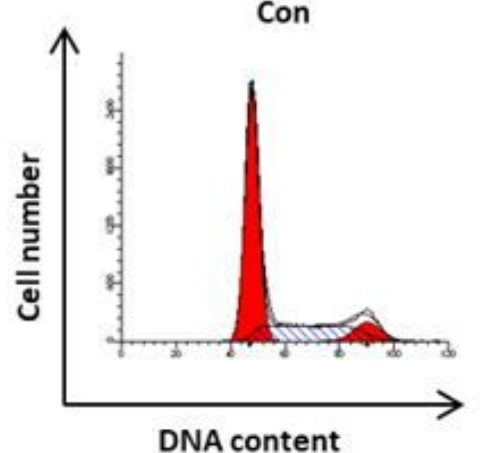

0.5

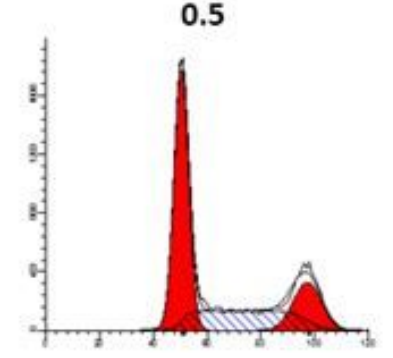

1.0

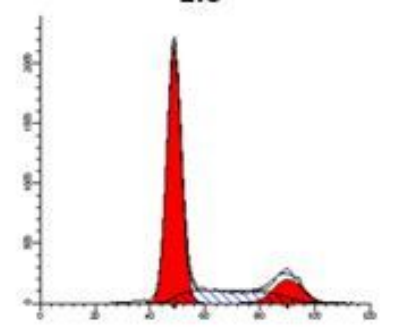

0.5

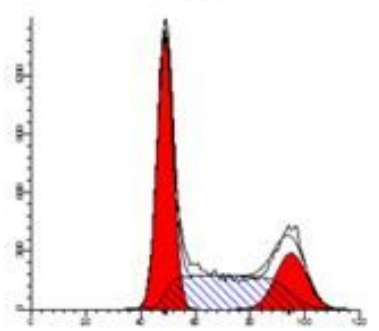

1.0

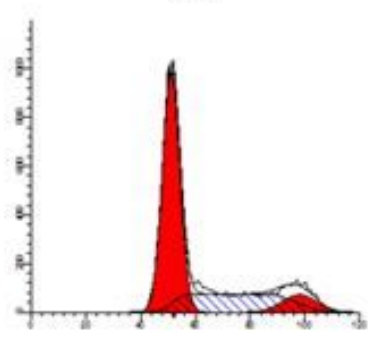

1.0

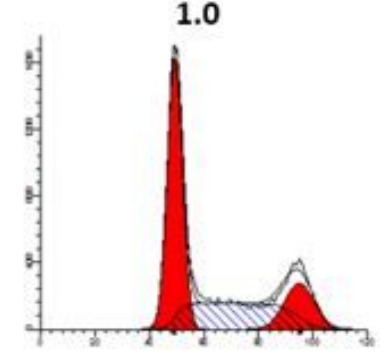

1.5

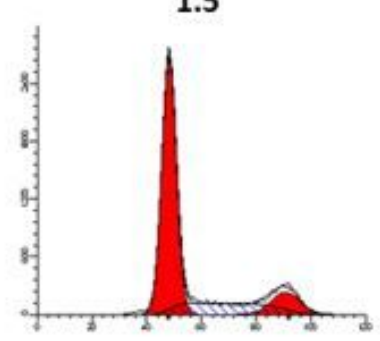

1.0

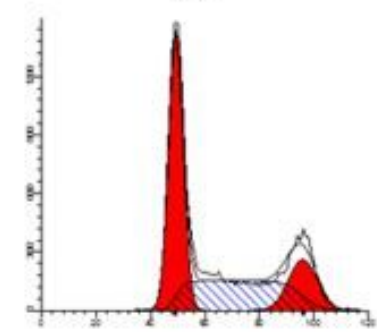

1.5

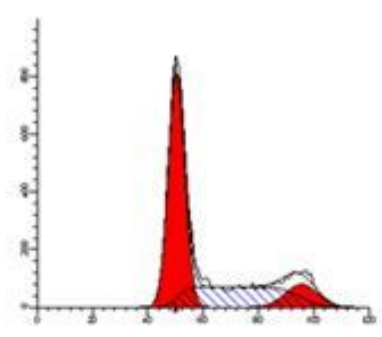

1.5

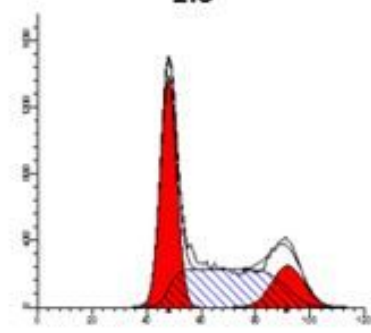

2.0

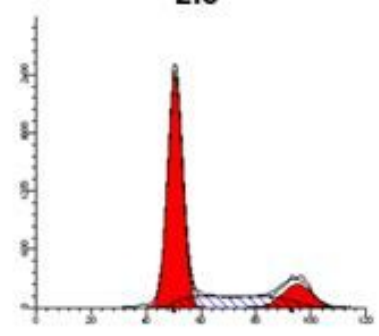

1.5

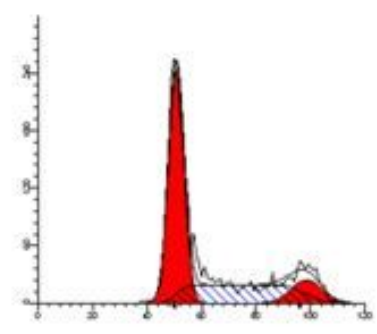

2.0

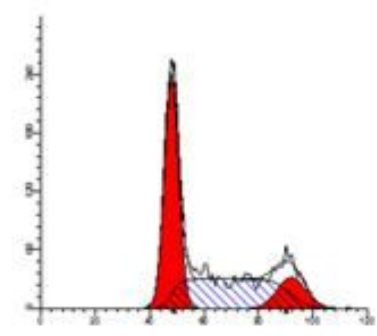

MCF-7

Cal-51

SKBR-3

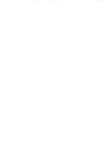

Figure 3

Effect of Ruanjian Sanjie (RJSJ) on the cell cycle in breast cancer cells. MDA-MB-231, MCF-7, Cal-51, and SKBR-3 cells were treated with various concentrations of RJSJ or a vehicle control for $48 \mathrm{~h}$. The cells were then collected, fixed, and analyzed via flow cytometry after staining with PI. The cell cycle distribution is presented as the mean $\pm \mathrm{SD}, * \mathrm{P}<0.05$ vs. control. 


\section{A}

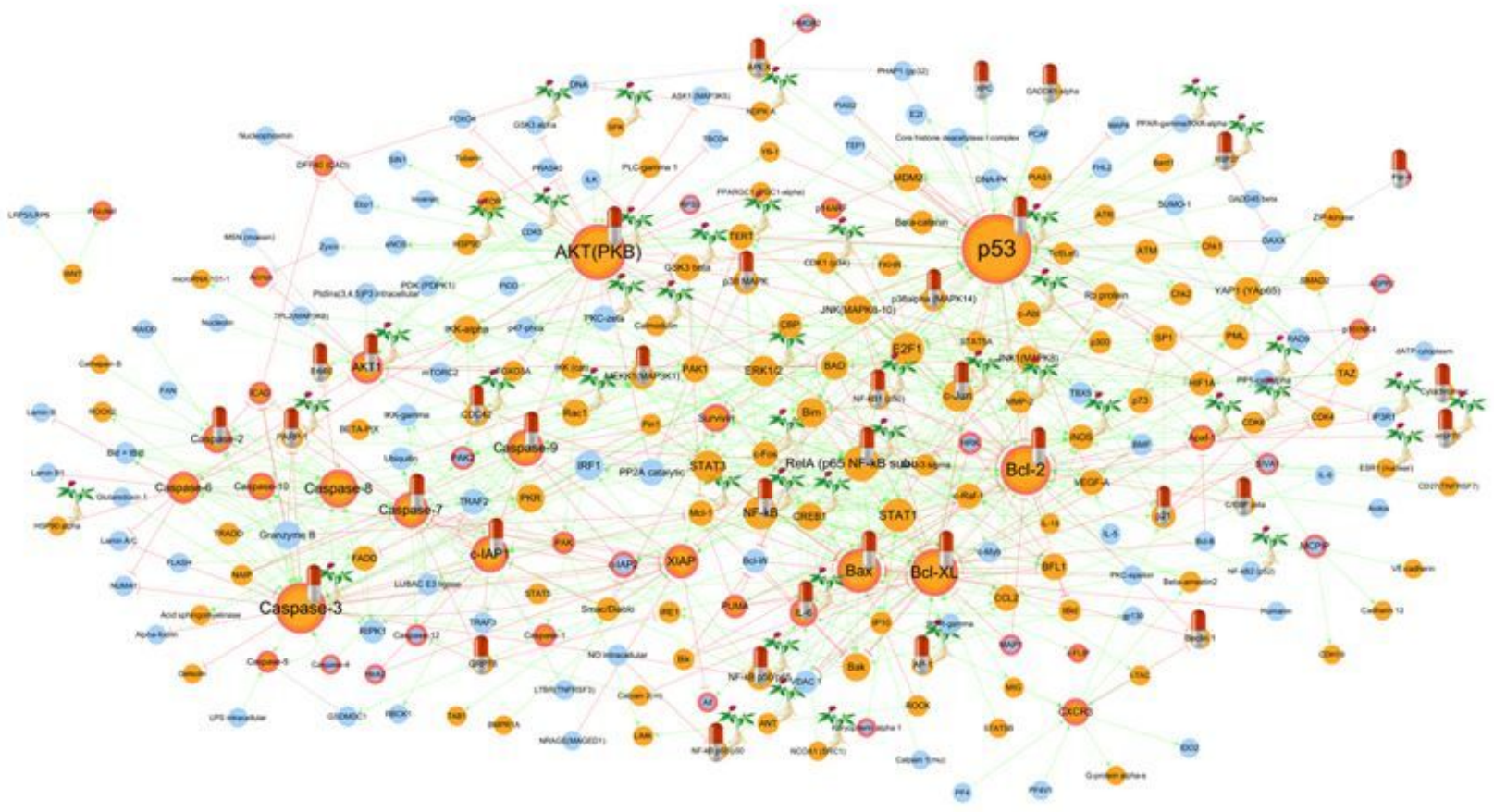

B

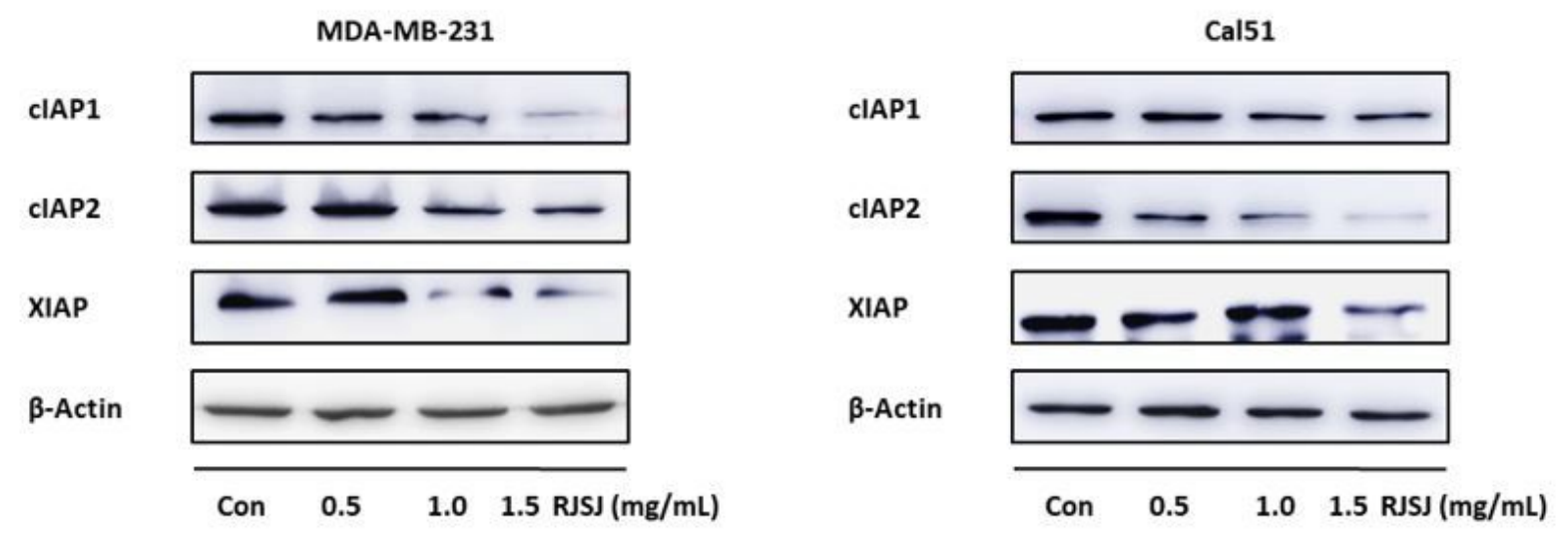

Figure 4

Ruanjian Sanjie (RJSJ) induces apoptosis by downregulating cellular inhibitor of apoptosis protein $1 / 2$ (cIAP1/2) and X-linked inhibitor of apoptosis protein (XIAP) in breast cancer cells. (A) "Integrated apoptosis module network of breast cancer" based on canonical apoptosis signaling pathways. There are 250 nodes and 692 edges in the network. Orange nodes represent breast cancer genes, blue nodes denote the intermediate nodes in the pathway, and nodes with a red circle represent the apoptosis genes. Nodes with a small sign of herb or capsule represent potential targets of RJSJ or carboplatin, respectively. Edges represent the interaction between nodes. There are three main types of interactions: activation (green lines), inhibition (red lines), and other (gray lines). The node size is proportional to the number of neighbor nodes. (B) RJSJ inhibited cIAP1/2 and XIAP expression. MDA-MB-231 and Cal-51 cells were treated with different concentrations of RJSJ for $48 \mathrm{~h}$, and cIAP1/2 and XIAP expression was detected using western blotting. $\beta$-Actin was used as a loading control. 
A

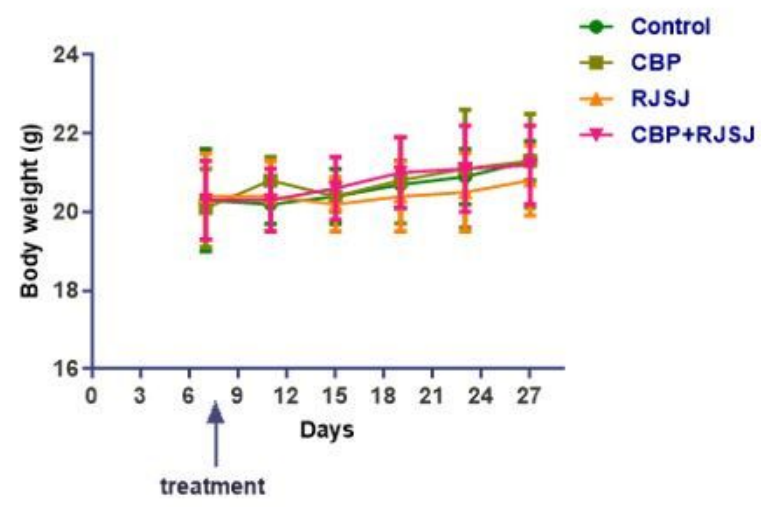

C

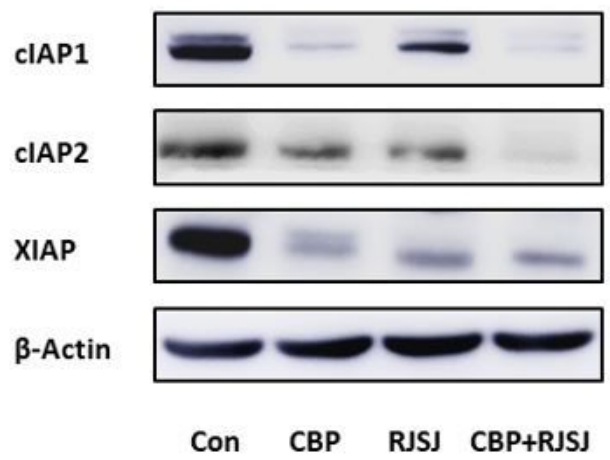

B

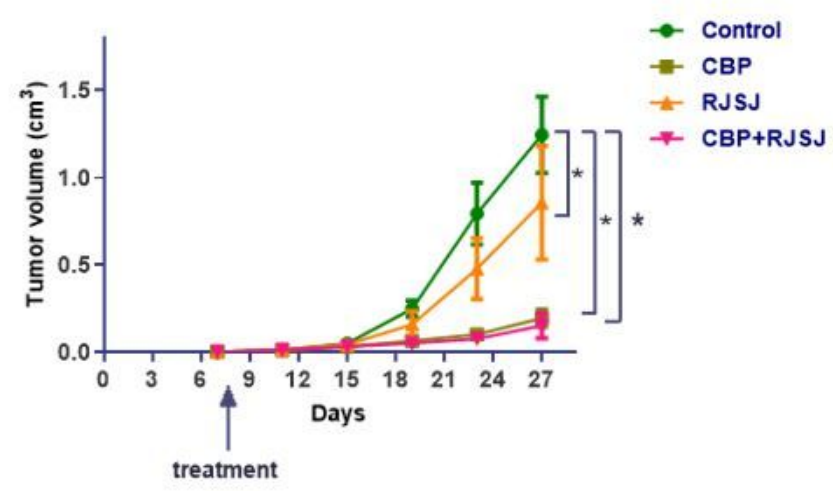

D

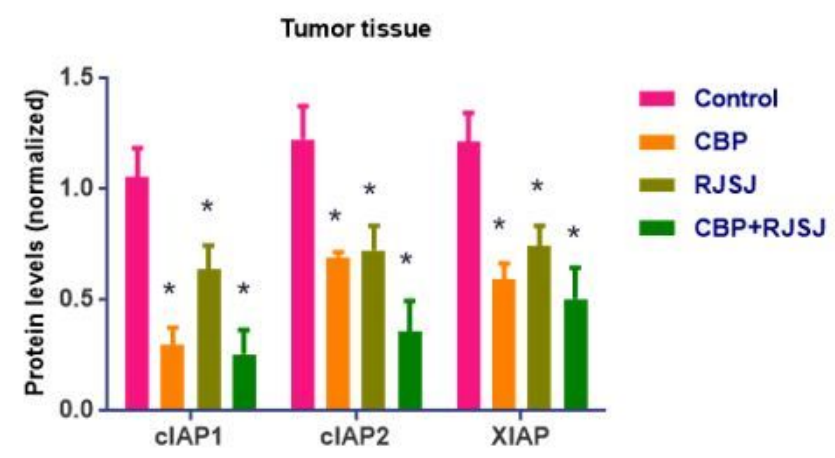

\section{Figure 5}

Ruanjian Sanjie (RJSJ) inhibits tumor growth in a nude mouse MDA-MB-231 tumor xenograft model. (A) Body weight and (B) tumor volume were measured in nude mice in each group. Data are presented as the mean \pm SD (six mice per group). (C) Protein was isolated from tumors, and cellular inhibitor of apoptosis protein 1/2 (CIAP1/2) and X-linked inhibitor of apoptosis protein (XIAP) expression was determined via western blotting. (D) Quantification of clAP1/2 and XIAP levels. Values are presented as mean \pm SD. ${ }^{*}<<$ 0.05 . 
(A)

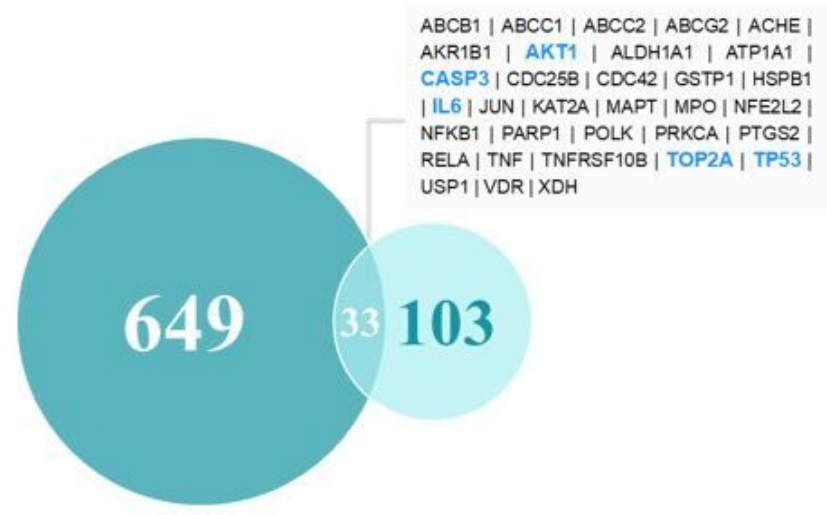

(C)

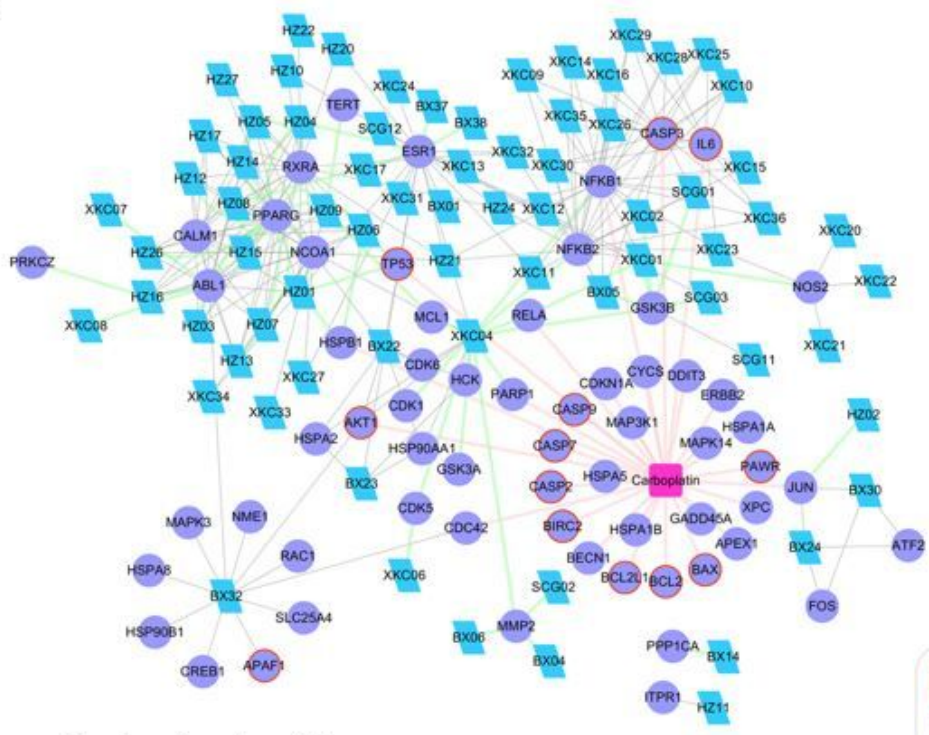

Number of nodes $=136$ Number of edges $=293$
(B)

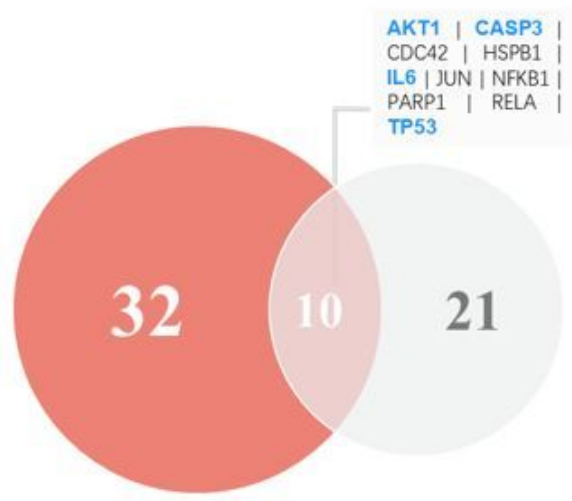

(D)

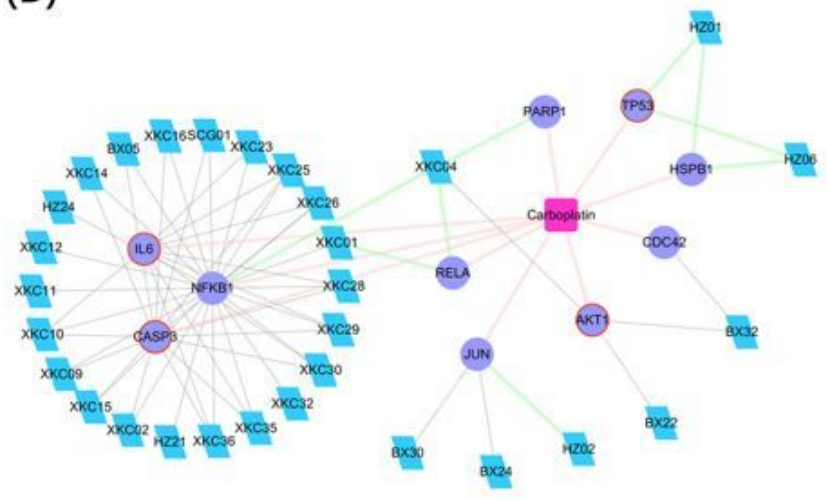

Number of nodes $=41$ Number of edges $=71$

Carboplatin __ Predicted compound-target relationship Targets Known compound-target relationship

Compounds Carboplatin-target relationship

\section{Figure 6}

Analysis of the synergistic effects of Ruanjian Sanjie (RJSJ) and carboplatin (CBP). (A) Comparison of target sets between RJSJ and CBP. (B) Comparison of targets mapped in the "integrated apoptosis module network of breast cancer" between RJSJ and CBP. (C) Relationship between compounds in RJSJ or CBP and their corresponding targets in the "integrated apoptosis module network of breast cancer." (D) The sub-network extracted from (C) based on the common targets regulated by RJSJ and CBP. Nodes with red circle are apoptosis-related genes.

\section{Supplementary Files}

This is a list of supplementary files associated with this preprint. Click to download.

- FigureS1.tif

- Figures2.tif 
- FigureS3.tif

- TableS1.xIsx

- TableS2.xIsx

- TableS3.xIsx

- TableS4.xIsx

- TableS5.xIsx

- Tables6.xIsx

- TableS7.xlsx

- TableS8.xlsx

- TableS9.xIsx

- TableS10.xlsx 